\title{
44
}

\section{Visual programming: an educational experience}

\author{
S. Rusnak \\ H. Schauer \\ A. Vaduva \\ University of Zurich \\ Switzerland
}

\begin{abstract}
This paper describes our educational experience in teaching programming techniques to first grade students. The introduction to basic programming constructs was made using a visual programming language called Prograph. The aim was to improve the understanding of programming concepts. In the second part of the course, advanced algorithms were introduced using the typical textual language Pascal. In this paper, we present the results of our investigation comparing the students attitude to these different programming styles. We found that almost all students with programming experience preferred Pascal as their favourite language, while students without any previous programming knowledge showed equal preference for both languages.
\end{abstract}

Main conference themes: informatics as study topic, methodologies

Educational areas: higher education

Study topics: computer science/informatics

Secondary keywords: algorithms, programming 


\section{INTRODUCTION}

Visual programming is a young, growing area of research. It uses pictures (icons, animation) and graphical representations in order to ease the task of programming. Pictures are supposed to be more powerful as means of communication and they support understanding and remembering [1]. Visual tools with limited applicability (e.g. medical diagnosis, scientific visualization, CASE tools) have already proved their usability and effectiveness for the common end-user. Direct manipulation interfaces [2] for operating systems are more and more replacing command-line interfaces. Domain specific visual programming languages gain popularity in the computer world (e.g. LabVIEW for controlling measuring instruments, VIVA [3] for image processing, PhonePro [4] - a telephony applications builder for creating automated, programmable voice-mail systems).

The state-of-the-art of general purpose visual programming languages only partially supports the claims of enhancing programmer's productivity and improving understandability. But the long term hope is to develop visual programming languages which "will provide a medium for expressing algorithms in ways which we at present cannot even imagine" and which "tomorrow's programmers would prefer to purely textual languages for their every-day tasks" [5]. Milestones in the development are the visual programming languages Prograph [6] and Serius [7]. In order to improve the current state of the visual programming feedback from users is needed. Emperical studies and experiences with general purpose visual languages help to analyze their advantages and drawbacks. Naturally, results of these studies cannot be always generalized. But these provide fresh impetus for new research.

We used a visual language to teach programming during the introductory computer science course at our university. Basically, this course consists of lectures and exercises in word processing, spreadsheet calculation, hypermedia and communication, followed by an introduction to object oriented programming [8].

It was our hypothesis that use of a visual programming language for the introduction of basic programming concepts would make the learning process easier. We also wanted to avoid the initial difficulties caused by teaching a textual language syntax. We assumed that nonprogrammers would be far more comfortable with visual programs than with a code written in a textual language. On the other hand we assumed that students with some programming experience would prefer the classical way of programming.

Our students mostly were absolute beginners; only a minority was more or less familiar with programming languages (Basic, Pascal, C). After an 
introduction to programming with Prograph, we asked the students in a first questionnaire about their attitude to visual programming.Then Pascal was taught and we asked the students to compare their experiences in both languages.

\section{Related work}

We refer to three studies which measure effectiveness of visual programming languages. In these studies small groups of subjects are analyzed using objectively measurable quantities; our results are based for the most part based on the (relative) opinions of a large number (196) of students.

The problematic nature of all these studies was synthesized in [9]: "Is one representation (i.e.. visual versus textual) better only for certain stages of learning, only for certain aspects of programming, or only for certain learners?"

A recent study [10] analyzes the construction of programs in a visual programming language, Forms $/ 3$, as compared with two textual languages: Pascal and a version of APL well suited for matrix manipulation. The study proved that vector and matrix manipulation problems were easier solved in the visual language. The solutions presented by 60 computer science students demonstrated the absolute superiority of the visual language.

While the previous experiment measures program construction in a restricted domain, our experiment was made with a general purpose language used for common beginner problems.

In [9] an empirical study of novices' program comprehension of a visual flowchart language is described in comparison with the Pascal language. The study investigates the speed (reaction time between question and response) and the accuracy with which the 23 subjects responded to questions about both languages. The results indicate that almost all of the subjects did understand more rapidly and more accurately the graphically represented program segments, as opposed to their textual equivalents.

In [11] the authors say that the value of a language structure (no matter if textual or visual) depends on the task. Therefore particular aspects of a language can support or hinder various programming tasks. The experiment was conducted on five subjects with some programming experience and also some experience in using LabView. The clear overall result was that the comprehension and design of graphical programs took longer than that of the textual ones. Nevertheless the experiment brought confirmation of an argument made in [9] that graphic representations of operators make them easier to recognize. The second important result of this experiment was the fact, that broader experience led to more flexible performance.

The same idea is emphasized in [12]. "Training and experience play a significant role in determining what is salient" when reading graphical representations. The authors supported this statement by a second experiment 
with 2 groups, novices and experts. Experts used more effective strategies than novices, who suffered from misreadings and confusion. Experts nearly always used text to guide the reading of a graphical representation. The conclusion was that the use of graphical notations requires experience, personal skill and training of both the creator and the reader to achieve the most effective communication.

It should be noted that the novices here were occasional programmers with programming experience from 5 to 20 years, who had used LabView for their work for at least six months. Our novices were absolute beginners, students without any programming experience and, partly, even without any intention to write programs in the future.

\section{Languages}

Prograph is a typical dataflow, object oriented visual programming environment and language. A program, called a universal method in Prograph, is described by an acyclic, directed graph in which nodes represent operations and arcs represent the paths over which data move between nodes. Every operation is a call to either a system defined (primitive) or a user defined method. Prograph methods are similar to functions and procedures in a textual language. Universal methods can be used as black boxes hiding all the details of their implementation and therefore are good mechanisms for introducing structure.

Pascal is a typical structured, imperative language, commonly used for learning programming. At our university we use THINK-Pascal, an object oriented version for the Macintosh. It is user-friendly: most of the syntax errors are detected during editing and the source code is automatically formatted ('pretty printing').

\section{Why Prograph?}

Firstly Prograph is one of the few commercially available general purpose visual programming languages. Prograph offers also, in our opinion, a lot of advantages for beginners in learning programming:

- Thanks to its special dataflow oriented architecture, variables appear only implicitly in the form of connections between methods and their interfaces.

- Executable dataflow graphs support development of mental images. Students can see how data are transferred between various program parts, they can also more easily understand the notion of interface and the need of input parameters for a procedure (Fig. 1).

- Prograph introduces lists as primitive data structures (c.f. Lisp). Therefore lists and list processing operations can be used in algorithms without problems. 
- The environment supports dynamic tracing of execution, reading and modifying values at runtime, and other user friendly functions.

- Last, but not least, we expected the students to have more fun and satisfaction working with a visual programming language.

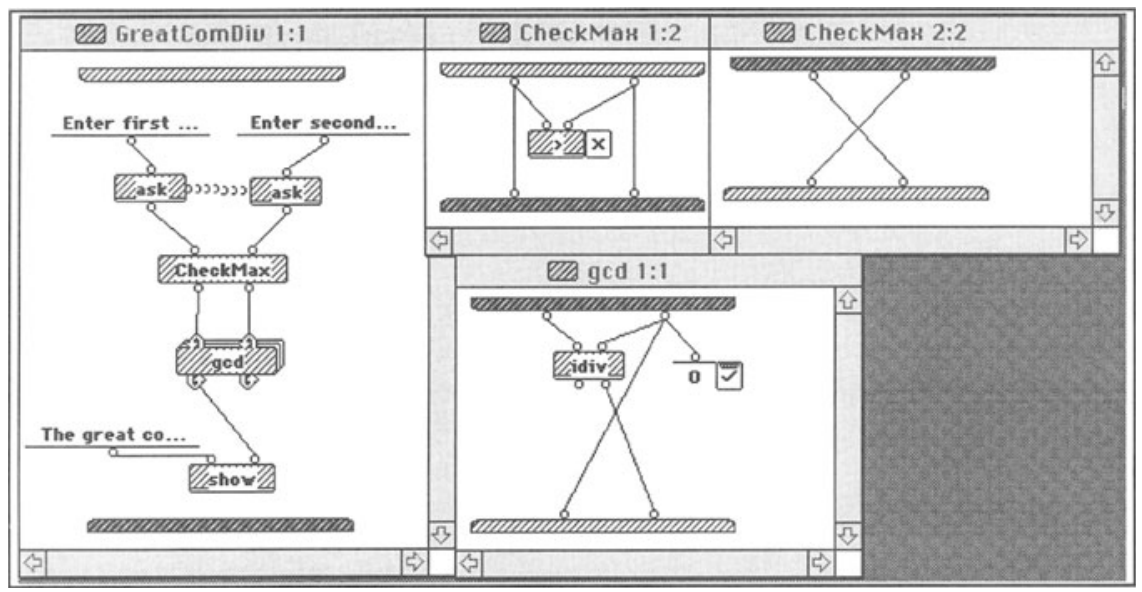

Fig. 1 A Prograph program to calculate the greatest common divisor of two numbers

\section{Participants}

The basic-level computer science course is compulsory for students in economy and informatics. The majority of participants will go on to attend advanced courses in either business management $(50 \%)$ or economics $(30 \%)$. Only a minority will choose computer science $(15 \%)$. The rest $(5 \%)$ are students in other subjects like mathematics, physics, biology and medical sciences. Of the 440 participants of the course 196 students answered our questionnaire.

First the motivation for attending the course. Multiple choice answers revealed the majority (46\%) to be indifferent ("actually, I am interested how to program") or little motivated (27\%): "I like to use computers for my work, but I do not wish to learn programming by myself". Great motivation ("I would like to solve programming problems by myself and therefore learn a new programming language") was shown by $18 \%$, while a few (9\%) answered: "I attend this course just because it is compulsory". In conclusion most students do not intend to become programmers, but use computers as end-users.

Secondly experience in programming. Most had none $(42 \%)$ or some $(42 \%)$; only a few (16\%) stated that they had a lot of experience (what ever this means at the age of 20). 
In the following, novices indicate those with none or some experience, experienced students those with a lot of experience.

\section{Lectures}

There were 8 lectures ( 2 hours each). First a general presentation of the notion of an algorithm, followed by an analysis of some structured programming constructs in the second and third lecture. All algorithms were illustrated with the visual programming language Prograph in dynamic representation. We assumed that dynamic, life execution would make concepts more understandable.

Explanations about the Prograph language and environment were kept to a minimum. Only the interpreter and a few functions of the environment were used for running the programs. We began with the traditional "Hello world" program and developed it step by step to a nice dialogue. For this purpose simple selection and repetition control were taught.

Following examples had to do with binary coding and decoding of decimals using iteration and recursion. Also an algorithm for the calculation of ' $x$ power $\mathrm{n}$ ' was shown, again using both iteration and recursion. Finally this algorithm was optimized by reducing the number of recursive steps.

In the questionnaire, we asked the students to choose from a list of opinions about these Prograph demonstrations. More choices were allowed. We compared answers of novices with the answers of more experienced students (Fig. 2).

Both novices and experienced students indicated that "Prograph demonstrations were a good way to represent introductory concepts". Novices held the opinion that "Prograph made the introduction to programming easier" and "made comprehension of presented algorithms easier", but only few (although some!) experienced students were of the same opinion. This is understandable: they already knew these concepts. The negative opinion "this did not help me particularly" is common in all groups, with an upward tendency from novices to experienced ones.

To test understanding of algorithms presented in the lecture, we asked "How did you understand the algorithms introduced in the lecture?" in both parts of the questionnaire: the Prograph and the Pascal part. There was a scale from " $1=$ not at all" to " $5=$ very well" to choose from. Again, the answers were related to the students programming experience (Fig. 3). 

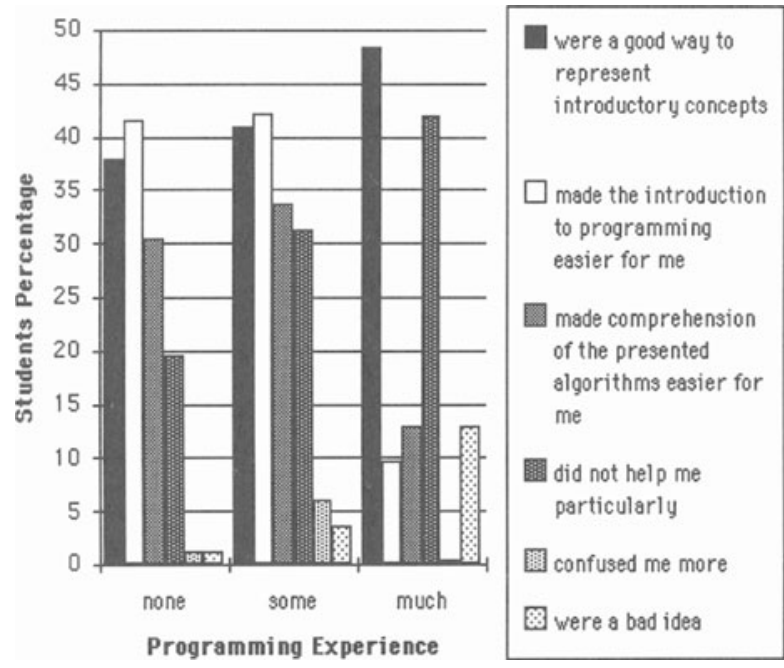

Fig.2 Students'opinions about introducing Prograph demonstrations related to their programming experience

Independent of what language they were using, the more experienced understood the algorithms presented in the lecture better (obvious). Only in the case of completely inexperienced programmers a slightly better understanding of algorithms presented in Prograph may be noted.

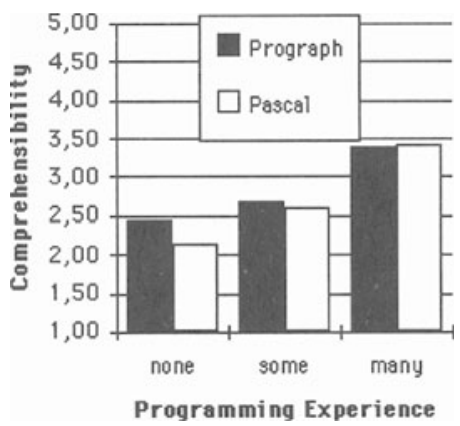

Fig. 3 Students' understanding of the algorithms presented in the lecture related to their programming experience 


\section{Lecture Experience}

With Prograph, structured constructs like sequence, iteration, recursion and abstraction can be well visualized. In particular, the concept of recursion is very difficult to explain in a textual programming language. In Prograph each instance of a recursive call can be visually identified with the help of environment features like showing method calls in a stack window and tracing execution. In the case of iteration, the need of an initial condition and a termination criterium is emphasized through the visual representation. But, unfortunately, the two elements are not presented in the same window, as if they do not belong together. The concept of selection is, in our opinion, not represented very intuitively either. The Prograph choice of displaying each branch of the case in a separate window reduces readability and makes it hard to keep a clear overview over the program.

\section{Exercises}

Exercises were offered for 2 hours a week. For these, students worked in groups of about 20 people in a computer lab, each group supervised by a teaching assistant. Every two weeks they received a new task to solve.

In the first programming exercise there were 2 problems to solve with Prograph: a simple string processing task for practising input-output operations and concatenation of strings; secondly a calculation of the greatest common divisor of two integers (Fig. 1).

The next exercise was to write a Pascal program which reads in a sequence of integers, calculates the maximum and displays this. In this way, simple input-output handling, iteration and selection were practised.

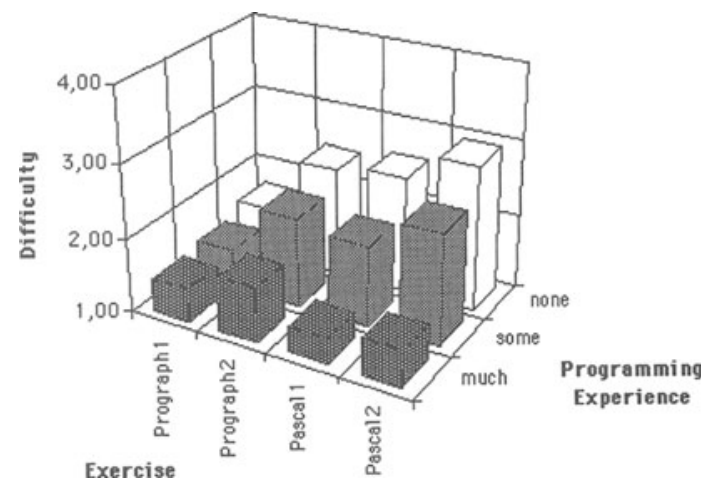

Fig. 4 Difficulty in solving programming tasks in Prograph and Pascal related to students' programming experience 


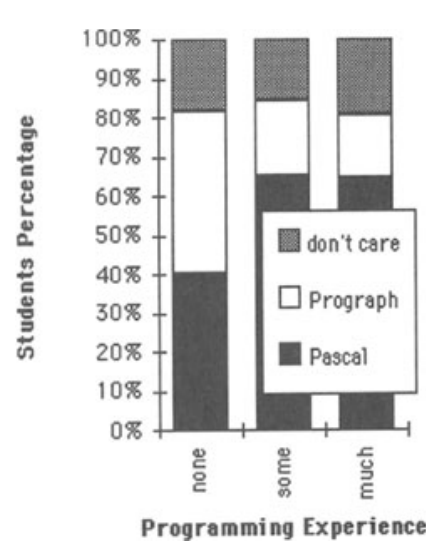

Fig. 5 (a) Language more convenient for introduction to programming

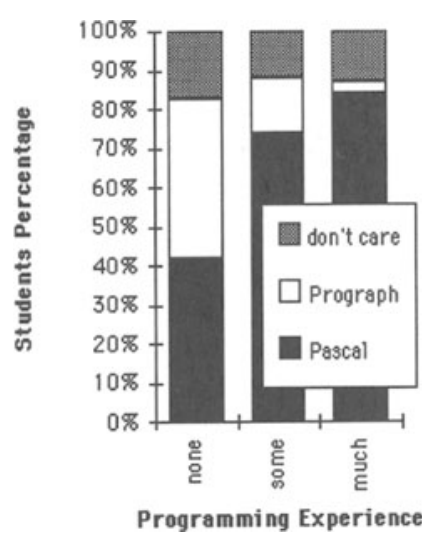

Fig. 5 (b) Language personally preferred for programming

In the last Pascal exercise, students had to save a sequence of integers in a data file, read the sequence from the file and present the data in a simple graph. They learned structuring into functions and procedures, file handling and simple graphical operations.

To compare students performance in both languages, we asked them about their difficulties solving the tasks in Prograph and Pascal. The difficulty scale in answer to the question "I was able to solve problem" was: $1=$ by myself immediately; 2 = after some initial difficulties by myself; $3=$ only with additional help from somebody else; $4=\mathrm{I}$ have not even tried to solve the problem.

Obviously the more experienced the students were, the less difficulties they generally had with all the exercises. Interesting results included the fact that experienced students seemed to have more difficulties with the Prograph exercises than with the Pascal ones. The reason for that will be the difficulty to rethink - to change one's. As the teaching assistants reported, the experienced ones also tried to influence the atmosphere with negative remarks about 'playing with Prograph'.

Novices do not show significantly greater difficulties in changing from a visualized to a textual way of programming. Comparing the two exercises Prograph 2 and Pascall, dealing with almost identical tasks, there is a big jump on the difficulty scale in the case of the experienced, but there is almost the same performance in the case of the inexperienced. 

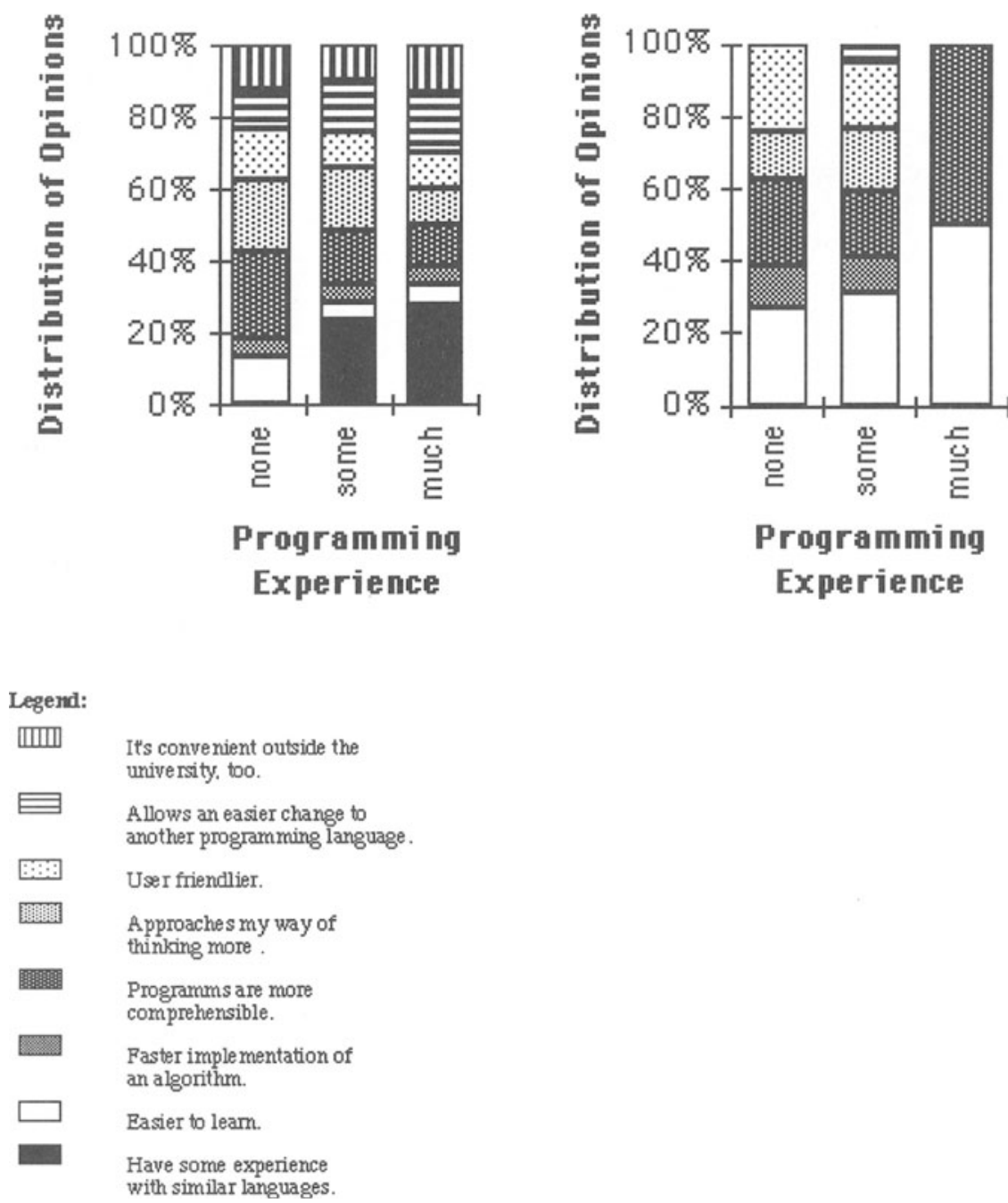

Fig. 6 (a) Reasons for choosing Pascal (b) Reasons for choosing Prograph related to programming experience

The last exercise, Pascal2, was judged by the students as the most difficult one. This however has nothing to do with the language, but with the complexity of 
the task. Therefore, we cannot conclude, that programming in Pascal is more difficult for students than in Prograph.

\section{Pascal versus Prograph}

In the last part of the questionnaire students had to answer the following questions: "Using which language do you think offers a better introduction to the subject of programming?" and "Which language did you personally like more for the use of programming?".

Among the group of novices Prograph and Pascal were chosen equallyboth for introduction and as personally preferred language. Among the more experienced an overwhelming majority preferred Pascal. Nevertheless, some of the experienced - although personally preferring Pascal-recognized Prograph as a convenient language for an introduction to programming.

Next, we asked the students to give some reasons for their choice in a multiple choice. One of the most important reasons for choosing Prograph is its "easiness to learn", while in the case of Pascal this was seldom mentioned. Similarly, the point "user friendlier" seems to play an important role for choosing Prograph instead of Pascal. However, nobody did choose Prograph because of "convenient outside the university, too" or "allows an easier change to another programming language", while this is one of the most important points for choosing Pascal (next to "have already experience with similar languages"). The reasons "reflects my way of thinking" and "programs are more comprehensible" were mentioned equally in both cases. Furthermore, there was a slightly higher percentage of students choosing Prograph because of "faster implementation of an algorithm".

\section{CONCLUDING REMARKS}

Introducing algorithms using Prograph proved to be very stimulating for student motivation and interest. In the exercises however, students had a lot of difficulties with the construction of Prograph programs. It seems that syntax problems were replaced by software operating problems (keyboard combinations for producing and linking special operations, controls).

Anyway, we were surprised about the improvement of student performance in Pascal programming compared to the year before. The reason might on the one hand be the introduction to general programming concepts using Prograph, on the other hand the repetition of the same programming task in another view. We used Prograph to make the starting easier and we think we reached our goal. 


\section{REFERENCES}

1 Shu, N. C. (1988) Visual Programming. New York: Van Nostrand Reinhold.

2 Shneiderman, B. (1983) Direct Manipulation: A Step Beyond Programming Language. Computer, August 89, pp. 57-69, IEEE Press.

3 Tanimoto, S. L. (1990) VIVA: a visual language for image processing. Journal of Visual Languages and Computing 1, pp. 127-139.

4 Ga Côté, R. (1994) Desktop Telephony. Develop your own automated phone applications with PhonePro, Byte, March.

5 Cilinert, E. P., Blattner, M. M. and Frerking, C.J. (1991) Visual Tools and Languages: Directions for the '90s. Proceedings 1991 IEEE Workshop on Visual Languages, Kobe, Japan, pp. 89-95.

6 C.ox, P.T., Giles, F.R. and Pietrzykowski, T. (1989) Prograph: a step towards liberating programming from textual conditioning. Proceedings 1989 IEEE. Workshop on Visual Languages, pp. 150-156.

7 Serius Corporation. (1992) Serius Programmer User's Guide.

8 Schauer, H. (1991) A first course in computer science at the University of Zurich. Education \& Computing 7, pp. 87-95.

9 Cunniff, N. and Taylor, R.P. (1987) Graphical vs. Textual Representation: An Empirical Study of Novices' Program Comprehension. Empirical Studies of Programmers: Second Workshop (eds Olson, G., Sheppard, S. and Soloway, E.), Ablex Publishing, Norwood, NJ.

10 Pandey, R. K. and Burnett, M. M. (1993) Is It Easier to Write Matrix Manipulation Programs Visually or Textually? An Empirical Study. Proceedings of the 1993 IEEE Symposium on Visual Languages, Bergen, Norway. 
11 Green, T.R.G., Petre, M. and Bellamy, R. (1991) Comprehensibility of Visual and Textual Programs: A Test of Superlativism Against the 'MatchMismatch' Conjecture. Empirical Studies of Programmers: Fourth Workshop (eds Koenemann-Belliveau, J. Moher, T. and Robertson, S.), Ablex Publishing, Norwood, NJ.

12 Petre, M. and Green, T.R.G. (1993) Learning to Read Graphics: Some Evidence that 'Seeing' an Information Display is an Acquired Skill. Journal of Visual Languages and Computing 4, pp. 55-70. 